NSF-ITP-99-133

hep-th/9911057

\title{
On the Hierarchy Between Non-Commutative and Ordinary Supersymmetric Yang-Mills
}

\author{
Akikazu Hashimoto \\ Institute for Theoretical Physics \\ University of California, Santa Barbara, CA 93106 \\ aki@itp.ucsb.edu \\ N. Itzhaki \\ Department of Physics \\ University of California, Santa Barbara, CA 93106 \\ sunny@physics.ucsb.edu
}

\begin{abstract}
Non-commutative supersymmetric Yang-Mills with rational $\Theta$ is dual to an ordinary supersymmetric Yang-Mills with a 't Hooft flux. It is believed that the simplest description is via the ordinary supersymmetric Yang-Mills. We claim, however, that the two descriptions form a hierarchy. The SYM description is the proper description in the ultra violet while the non-commutative description takes over in the infra red.
\end{abstract}




\section{Introduction}

It is well known that a non-commutative supersymmetric Yang-Mills theory (NCSYM) with non-commutativity scale $\Delta$ on a torus of size $\Sigma$ is equivalent to an ordinary supersymmetric Yang-Mills theory (OSYM) with a magnetic flux if the dimensionless non-commutativity parameter $\Theta=\Delta^{2} / \Sigma^{2}$ is a rational number. The equivalence between the NCSYM and the OSYM can be understood naturally as arising from the T-duality of the underlying string theory $[1,2]$. The structure of T-duality survives the decoupling limit and can be identified as the Morita equivalence of the NCSYM. The precise relation between T-duality and Morita equivalence was studied extensively $[3,4,5,6,7,8,9,10,11,12,13,14,15]$.

Whenever a theory admits dual descriptions, there is always a unique canonical description that is in some sense natural. For example, in the case of duality relation which exchanges strong and weak couplings, it is more natural to describe the physics in terms of the weakly coupled theory. This is not a useful criterion for our purposes since Morita equivalence is not a strong-weak duality but rather a local-nonlocal duality. That is, it takes a local excitation of one description to a non-local excitation of the dual description. This suggests that the natural description is the one which is most local.

In light of this criterion, one might be inclined to conclude that the OSYM description is the ultimately local and therefore the most natural description of this theory. In that case, one can forget all together about the NCSYM with rational $\Theta$. However, in the presence of the magnetic flux, there are very light excitations, the electric fluxes, which are highly nonlocal. The locality of the OSYM description breaks down when these non-local excitations start to dominate the dynamics, and this happens precisely in the infra red at the energies of the order of the volume of the torus.

The primary goal of this paper is to demonstrate that the dynamics of these light nonlocal degrees of freedom of the OSYM admits a natural local description in terms of the NCSYM. In other words, the NCSYM takes over as the natural local description in the infra red when the locality of the OSYM breaks down due to finite size and the magnetic flux. This is the sense in which the NCSYM description is physically significant and should therefore not be dismissed even if the theory is equivalent to some OSYM by duality. NCSYM and OSYM are the components in the hierarchy of the phases of the same theory.

The paper is organized as follows. In section 2 we describe the OSYM-NCSYM hierarchy using weakly coupled field theory arguments. We also show how similar structures emerge from the supergravity description of these theories in the limit of large 't Hooft coupling. Concluding remarks and applications are presented in section 3. 


\section{NCSYM as the low energy limit of OSYM}

In this section, we will show how different Morita equivalent theories form a hierarchy of phases, and that for any given energies, there is a unique simple/natural description.

We start with the simplest case of NCSYM with $\Theta=1 / s$ for some integer $s$. There are two natural Morita equivalent descriptions of this theory: the NCSYM description in the infra red and the OSYM in the ultra violet. In section 2.1 we show this for a weakly coupled theory while in section 2.2 we obtain the same result for the strongly coupled theory using the supergravity description.

The general rational case $\Theta=r / s$ is considered in section 2.3. It is shown that as we increase the energy, the non-commutativity scale of the natural description decreases until we end up with some OSYM description in the deep ultra violet. We also comment briefly on the irrational case.

\subsection{NCSYM with $\Theta=1 / s$}

Consider a $U(N)$ NCSYM with coupling constant $g_{Y M}$ and vanishing magnetic flux $(m=0)$ on a torus of size $\Sigma$ with $\Theta=1 / s$ for some integer $s \gg 1$. This theory is equivalent to an ordinary SYM with

$$
\tilde{N}=s N, \quad \tilde{\Sigma}=\frac{\Sigma}{s}, \quad \tilde{g}_{Y M}^{2}=\frac{g_{Y M}^{2}}{s}, \quad \tilde{m}=N
$$

The relevant properties of the T-duality (Morita equivalence) used to derive this relation are summarized in the appendix.

It may seem strange that a theory defined on a torus whose volume is smaller by a factor of $1 / s^{2} \ll 1$ can be equivalent to a NCSYM on a much larger volume $\Sigma^{2}$, since the momentum modes in the ordinary theory have a gap of the order $1 / \tilde{\Sigma}=s / \Sigma$ while the momentum modes in the non-commutative description have a gap of the order $1 / \Sigma$. However, the fact that the background has a non-vanishing magnetic flux $\tilde{m}$ (or a twisted boundary condition [16]) plays a significant role here. In a presence of such a twist, the energies for the excitations corresponding to the electric fluxes winding around the torus are quantized in the units of

$$
\frac{\operatorname{GCD}(\tilde{m}, \tilde{N})}{\tilde{N}} \frac{1}{\tilde{\Sigma}}=\frac{1}{s \tilde{\Sigma}} .
$$

This was shown in [17] by studying the small fluctuation of gauge fields in the 't Hooft flux background [16] and can also be seen from the string theory considerations [18]. This can also be thought of as a realization of the mechanism of [19]. In this way, the OSYM in a twisted background and the NCSYM can have the same physical content. 
We will now argue that the NCSYM and the OSYM descriptions provide a natural description of the dynamics in a non-overlapping range of energies (see figure 1). Consider first the NCSYM. The range of energies where this description is natural is bounded both in the ultra violet and in the infra red. The infra red bound is the usual one which appears also for local theories. Namely, by lowering the energies to be of order $1 / \Sigma$, we reach energies of the order of the mass gap of the momentum modes and the effective description is in terms of the 2D OSYM.

The bound in the ultra violet is more interesting because ordinarily, the finite size do not affect the ultra violet. This is not the case with theories on non-commutative spaces. By going to higher energies, one might succeed in making the Compton wavelength $\Delta x_{2}=1 / E$ along, say, the 2 direction to be small. This will cause the size of the excitations to grow in the 3-direction in keeping with the non-commutativity $\Delta x_{2} \Delta x_{3}>\Delta^{2}$. Since $\Delta x_{3}$ is bounded by the size of the box $\Sigma$, we find that $E$ must be bounded above by $\Sigma / \Delta^{2}$ in order to keep the excitations localized. Combining these observations, we learn that the physics of the NCSYM is natural in the range of energies

$$
\frac{1}{\Sigma}<E<\frac{\Sigma}{\Delta^{2}}=\frac{s}{\Sigma}
$$

It is important to emphasize that at the fundamental level the NCSYM description does not break down above that scale. It is just becoming very complicated because the relevant excitations are not localized relative to the size of the torus.

Let us now consider the OSYM. Since this theory is an ordinary conformal field theory, one can expect this description to remain valid at arbitrarily high energies. However, on the infra red side, the momentum modes have a gap of the order of $1 / \tilde{\Sigma}$. Therefore, the range of naturalness of the OSYM description is bounded in the infra red by $E=1 / \tilde{\Sigma}=s / \Sigma$. Below this energy, we have a two dimensional OSYM in the absence of the magnetic flux. In the presence of the magnetic charge, however, there are excitations which are much lighter than the gap of the momentum modes, $1 / \tilde{\Sigma}$. These are the electric fluxes ${ }^{1}$ whose energies are quantized in the units of $1 / \Sigma$. Since the Compton wave lengths of the states with such energies are much larger than the size of the torus, these excitations are highly non-local and hence it is very hard to describe their dynamics in terms of the OSYM. In terms of the dual NCSYM description, these excitations are nothing but the momentum modes (see appendix and $[11,14])$ whose dynamics is much simpler.

It should be emphasized that the physics of the cross-over on the infra red side is different between the twisted SYM and the untwisted SYM. In the untwisted SYM, the cross-over

\footnotetext{
${ }^{1}$ At an intuitive level, the electric fluxes behave differently in the magnetic background because they also carry momentum due to the Pointing vector $\vec{P} \sim \vec{B} \times \vec{E}$. See the appendix and $[11,17]$ for a more detailed discussion.
} 


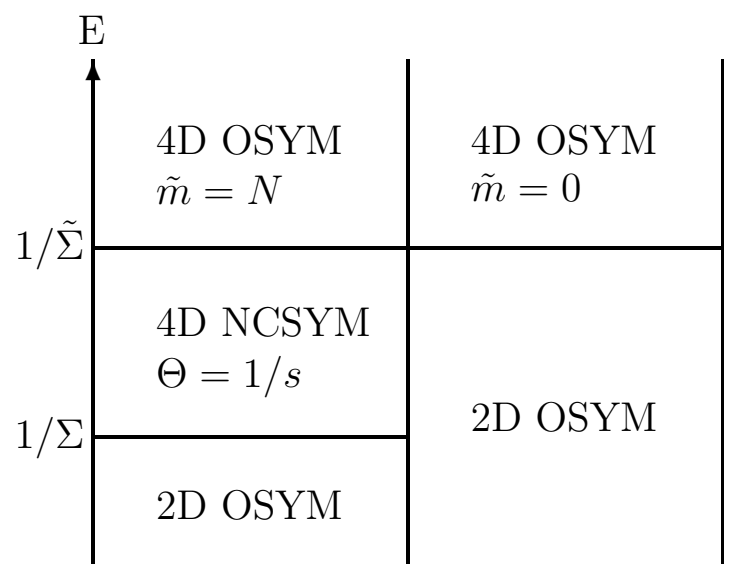

Figure 1: The phases of SYM on $R^{2} \times T^{2}$ : In the absence of a magnetic flux the theory flows to a two dimensional theory at energies below $1 / \tilde{\Sigma}$. In the presence of a magnetic flux there are excitations which are lighter than $1 / \tilde{\Sigma}$. The dynamics of these excitations is described by the NCSYM in four dimensions. Only deeper in the infra red the theory becomes two dimensional.

in the infra red is due to the freezing of all but the zero momentum modes, whereas in the twisted SYM, there are additional degrees of freedom due to the electric fluxes. The dynamics of these degrees of freedom is given not in terms of a dimensionally reduced theory, but by the 4D NCSYM.

It should also be emphasized that the twisted SYM, the NCSYM, and the dimensionally reduced SYM descriptions are natural in their respective non-overlapping range of energies, but they combine to cover the full range of energies.

\subsection{Large 't Hooft coupling and supergravity}

In the previous subsection, we assumed that the theory is weakly coupled to argue that the duality between the NCSYM and the OSYM gives rise to a hierarchy of phases. We will now show that a similar hierarchy appears also in the strongly coupled theory. This suggests that the structure of NCSYM-OSYM hierarchy is not sensitive to the value of the coupling constant.

The supergravity solution which is dual to the NCSYM with large 't Hooft coupling is $[20,21]$

$$
\begin{aligned}
d s^{2} & =\alpha^{\prime}\left\{\frac{U^{2}}{\sqrt{\lambda}}\left(-d t^{2}+d x_{1}^{2}\right)+\frac{\sqrt{\lambda} U^{2}}{\lambda+U^{4} \Delta^{4}}\left(d x_{2}^{2}+d x_{3}^{2}\right)+\frac{\sqrt{\lambda}}{U^{2}} d U^{2}+\sqrt{\lambda} d \Omega_{5}^{2}\right\} \\
e^{\phi} & =\frac{\lambda}{4 \pi N} \sqrt{\frac{\lambda}{\lambda+\Delta^{4} U^{4}}}, \quad B_{23}=-\frac{\alpha^{\prime} \Delta^{2} U^{4}}{\lambda+\Delta^{4} U^{4}}
\end{aligned}
$$


with periodicities $x_{2} \sim x_{2}+2 \pi \Sigma$ and $x_{3} \sim x_{3}+2 \pi \Sigma$. In the infinite volume limit $(\Sigma \rightarrow \infty)$, the solution can be trusted everywhere for large $N$ and $\lambda$. For finite $\Sigma$, the supergravity description breaks down at the ultra violet since the size of the torus is shrinking. This implies that a different T-dual description takes over. The transition occurs when the invariant size of the torus is of the order of the string length, so that the momentum modes are no longer the lightest modes. This happens at

$$
U=\frac{\lambda^{1 / 4} \Sigma}{\Delta^{2}} .
$$

Similarly, the size of the torus becomes small when $U$ gets smaller than $\lambda^{1 / 4} / \Sigma$. This implies, using the UV/IR relation [22], that the range of energies for which the supergravity description of the NCSYM at large 't Hooft is valid is

$$
\frac{1}{\lambda^{1 / 4}} \frac{1}{\Sigma}<E<\frac{1}{\lambda^{1 / 4}} \frac{\Sigma}{\Delta^{2}}
$$

This has basically the same form as (2.3) up to the factor of $\lambda^{1 / 4}$ which is a strong coupling effect.

Outside this range of energies, some other T-dual description is more natural. In the infra red the proper description is via the near horizon limit of D1-branes [23]. This is just the supergravity realization of the fact that the theory flows in the infra red to a theory in two dimensions. This transition, which involves a Gregory-Laflamme transition [24, 25] in addition to the T-duality, was discussed in [26, 27, 28] and will not elaborated on further here.

The more interesting transition for our discussion is the one in the ultra violet. The way T-duality acts on the supergravity solution is the following [29]. Define

$$
\rho=\frac{\Sigma^{2}}{\alpha^{\prime}}\left(B_{23}+i \sqrt{G_{22} G_{33}}\right)
$$

and act by an element of $S L(2, Z)$ according to

$$
\tilde{\rho}=\frac{a \rho+b}{c \rho+d}
$$

The dual $B$-field and the dual volume can be extracted from the real and the imaginary parts of $\tilde{\rho}$, respectively.

$$
\begin{aligned}
& b_{23}=\frac{\tilde{\Sigma}^{2}}{\alpha^{\prime}} \tilde{B}_{23}=\operatorname{Im}(\tilde{\rho})=\frac{b d \lambda+\left(b \Delta^{2}+a \Sigma^{2}\right)\left(d \Delta^{2}+c \Sigma^{2}\right) U^{4}}{d^{2} \lambda+\left(d \Delta^{2}+c \Sigma^{2}\right)^{2} U^{4}} \\
& v_{23}=\frac{\sqrt{\lambda} \Sigma^{2} U^{2}}{\alpha^{\prime}}=\operatorname{Re}(\tilde{\rho})=\frac{\sqrt{d^{2} \lambda+\left(d \Delta^{2}+c \Sigma^{2}\right)^{2} U^{4}}}{}
\end{aligned}
$$




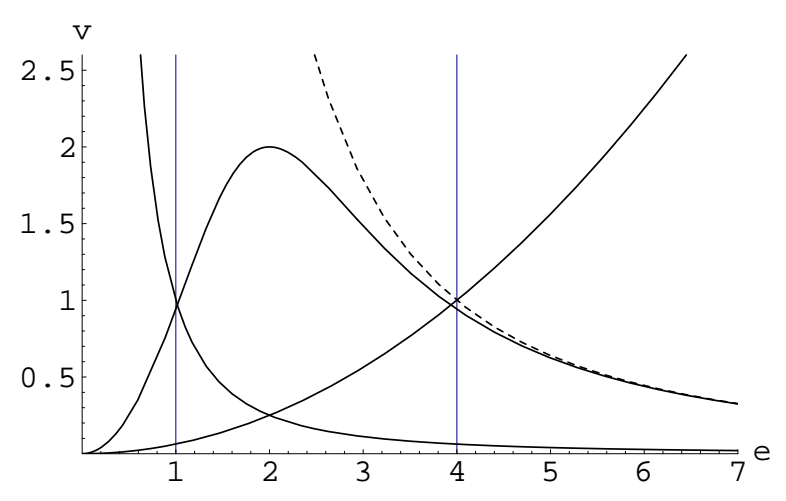

Figure 2: The different phases from the supergravity point of view. Each of the curves in corresponds to the volume of the torus in the supergravity solution as a function of $e=U / \lambda^{1 / 4}$ where we absorbed the factor of $\lambda^{1 / 4}$ into the definition of $e$ to account for the effect of strong coupling. We see that there are, like in the weakly coupled case, three "phases," corresponding to the dimensionally reduced SYM, the NCSYM, and the twisted OSYM. This is to be compared against the case with $\tilde{m}=0$, which we illustrated in the same figure using the dotted line. Here, there are only two "phases," corresponding to the dimensionally reduced SYM and the OSYM.

It is clear from (2.9) that generically, $v_{23}$ will always be small for sufficiently large $U$. In the case where $\Theta=\Delta^{2} / \Sigma^{2}=1 / s$, however, the coefficient of the term in the denominator of (2.9) proportional to $U^{4}$ can be set to zero by choosing $c=-1, d=s$. This is the same element of $S L(2, Z)$ which mapped the NCSYM to the OSYM in the previous subsection, and gives rise to the following background

$$
\begin{aligned}
d s^{2} & =\alpha^{\prime}\left\{\frac{U^{2}}{\sqrt{\lambda}}\left(-d t^{2}+d x_{1}^{2}+d x_{2}^{2}+d x_{3}^{2}\right)+\frac{\sqrt{\lambda}}{U^{2}} d U^{2}+\sqrt{\lambda} d \Omega_{5}^{2}\right\} \\
e^{\phi} & =\frac{\lambda}{4 \pi s N}, \quad B_{23}=-\frac{1}{s} \frac{\alpha^{\prime}}{\tilde{\Sigma}^{2}}
\end{aligned}
$$

with periodicities $x_{2} \sim x_{2}+2 \pi \tilde{\Sigma}$ and $x_{3} \sim x_{3}+2 \pi \tilde{\Sigma}$. The background geometry is exactly $A d S_{5} \times S_{5}$ which implies [30] that the theory is OSYM in 4D. The presence of the constant background $B$-field gives rise to the twist in the corresponding boundary theory. In particular, the constant $B$-field modifies the spectrum of closed string winding modes and not of the momentum modes, just as the twist modifies the spectrum of the electric fluxes and not of the local momentum modes. The radius of $S^{5}$ remained the same as (2.4). On the other hand, the number of D3-branes is multiplied by a factor of $s$, which can be seen either from the form of the dilaton or the form of the RR field background which can be found in [21]. The size of the torus, on the other hand, is smaller by a factor of $s$. All these features are in agreement with the field theory results of equation (2.1) which relate the NCSYM and the OSYM. 


\subsection{General $\Theta$}

In light of the analysis in the previous subsection for the case when $\Theta=1 / s$, it is straight forward to understand what happens in the case of a more general $\Theta$. Consider first the case where $\Theta=r / s$ is some rational number. Just as before, the NCSYM description is expected to be the natural description in the range of energies

$$
\frac{1}{\Sigma}<E<\frac{\Sigma}{\Delta^{2}}=\frac{s}{r} \frac{1}{\Sigma}
$$

and the dimensionally reduced description takes over for $E<1 / \Sigma$. On the other hand, the OSYM description is only valid at energies above $E=s / \Sigma$, and for $r>1$, there is a gap between $s / r \Sigma$ and $s / \Sigma$. Therefore, there must be some other Morita equivalent description which is valid in this intermediate regime. Indeed, it is easy to see that for every energy scale $E$, there is one and only one Morita equivalent description which satisfies

$$
\frac{1}{\tilde{\Sigma}}<E<\frac{\tilde{\Sigma}}{\tilde{\Delta}^{2}}
$$

It is straight forward to search for the appropriate $S L(2, Z)$ dual description using the relevant equations given in the appendix. For example, for $\Theta=13 / 47$ we find the following chain of natural descriptions ${ }^{2}$

\begin{tabular}{lcl}
$\tilde{\Delta}^{2}=\frac{13}{47} \Sigma^{2}$ & $\frac{1}{\Sigma}<E<\frac{47}{13} \frac{1}{\Sigma}$ & $M=\left(\begin{array}{cc}1 & 0 \\
0 & 1\end{array}\right)$ \\
\hline$\tilde{\Delta}^{2}=\frac{65}{2209} \Sigma^{2}$ & $\frac{47}{13} \frac{1}{\Sigma}<E<\frac{47}{5} \frac{1}{\Sigma}$ & $M=\left(\begin{array}{cc}0 & 1 \\
-1 & 4\end{array}\right)$ \\
\hline$\tilde{\Delta}^{2}=\frac{10}{2209} \Sigma^{2}$ & $\frac{47}{5} \frac{1}{\Sigma}<E<\frac{47}{2} \frac{1}{\Sigma}$ & $M=\left(\begin{array}{cc}-1 & 4 \\
-3 & 11\end{array}\right)$ \\
\hline$\tilde{\Delta}^{2}=\frac{2}{2209} \Sigma^{2}$ & $\frac{47}{2} \frac{1}{\Sigma}<E<\frac{47}{\Sigma}$ & $M=\left(\begin{array}{cc}-3 & 11 \\
-8 & 29\end{array}\right)$ \\
\hline$\tilde{\Delta}^{2}=0$ & $\frac{47}{\Sigma}<E$ & $M=\left(\begin{array}{cc}-8 & 29 \\
-13 & 47\end{array}\right)$
\end{tabular}

where $M$ is an element of $S L(2, Z)$ defining the Morita dual relative to the original theory with $\Theta=13 / 47$.

Once the case of general rational $\Theta$ is understood, it is not difficult to see what happens in the case of irrational $\Theta$. Since for irrational $\Theta$ we can never reach the $\tilde{\Theta}=0$ theory by finite action of $S L(2, Z)$, the chain of Morita equivalent theories will not terminate but continue indefinitely. For example, for $\Theta=\gamma$ where $\gamma \approx 0.58$ is the Euler's constant, we find

\footnotetext{
${ }^{2}$ In the following, we drop the dimensionally reduced SYM phase for brevity.
} 
the following chain of natural Morita equivalent descriptions.

\begin{tabular}{ccc}
$\tilde{\Delta}^{2}=0.58 \Sigma^{2}$ & $\frac{1}{\Sigma}<E<1.7 \frac{1}{\Sigma}$ & $M=\left(\begin{array}{cc}1 & 0 \\
0 & 1\end{array}\right)$ \\
\hline$\tilde{\Delta}^{2}=0.089 \Sigma^{2}$ & $1.7 \frac{1}{\Sigma}<E<6.5 \frac{1}{\Sigma}$ & $M=\left(\begin{array}{cc}0 & 1 \\
-1 & 2\end{array}\right)$ \\
\hline$\tilde{\Delta}^{2}=0.0063 \Sigma^{2}$ & $6.5 \frac{1}{\Sigma}<E<24.7 \frac{1}{\Sigma}$ & $M=\left(\begin{array}{cc}-1 & 2 \\
-4 & 7\end{array}\right)$ \\
\hline$\tilde{\Delta}^{2}=0.0003 \Sigma^{2}$ & $24.7 \frac{1}{\Sigma}<E<131.5 \frac{1}{\Sigma}$ & $M=\left(\begin{array}{cc}-4 & 7 \\
-15 & 26\end{array}\right)$ \\
\hline$\vdots$ & $\vdots$ & $\vdots$
\end{tabular}

Let us pause and comment about the general structure of these chains of theories. First, the parameters of two adjacent members of this chain should satisfy an inequality

$$
\frac{1}{\Sigma}<\frac{\Sigma}{\Delta^{2}}=\frac{1}{\tilde{\Sigma}}<\frac{\tilde{\Sigma}}{\tilde{\Delta}^{2}} .
$$

From this, it follows immediately that

$$
\tilde{\Sigma}<\Sigma, \quad \tilde{\Delta}<\Delta
$$

which states that both the non-commutativity and the volume decreases monotonically along the chain. Second, since all of these theories are equivalent to one another at the microscopic level, they should all have the same thermodynamic properties. Therefore, the relation between the entropy $S$ and the temperature $T$

$$
S \sim \tilde{N}_{\Theta}^{2} \tilde{\Sigma}^{2} R T^{3}, \quad \tilde{N}_{\Theta}=\tilde{N}-\tilde{\Theta} \tilde{m},
$$

must be invariant under the $S L(2, Z)$ duality group, which we verify in the appendix. Here, $R$ denotes the compactification radius in the $x_{1}$ direction, which we take to be much larger then $\Sigma$.

The same basic structure can be seen also in the supergravity description of the same model with large 't Hooft coupling. In figures 3.a and 3.b, we plot $v_{23}(e)$ for the chain of natural Morita equivalent theories (2.13) and (2.14) with $\Theta=13 / 47$ and $\Theta=\gamma \approx 0.58$, respectively. The vertical lines delineate the range of energies tabulated in (2.13) and (2.14). The left most peak in figures 3.a and 3.b illustrate the dependence of the torus size as a function of $e$ of the supergravity background corresponding to the NCSYM (2.4). Each successive peaks correspond to the supergravity background of some Morita dual. A given supergravity description is natural when $v(e)$ is of order one or greater. Therefore, we find that the chain of natural Morita equivalent theories (2.13) and (2.14) is also natural from the point of view of supergravity. If $\Theta$ is rational, eventually we will encounter $v(e)$ which grows like $e^{2}$ indefinitely (2.10). This is the right most curve illustrated in figure 3.a and corresponds to the OSYM. For irrational $\Theta$ like the one illustrated in figure 3.b, new peaks will appear indefinitely for arbitrarily large $e$. 


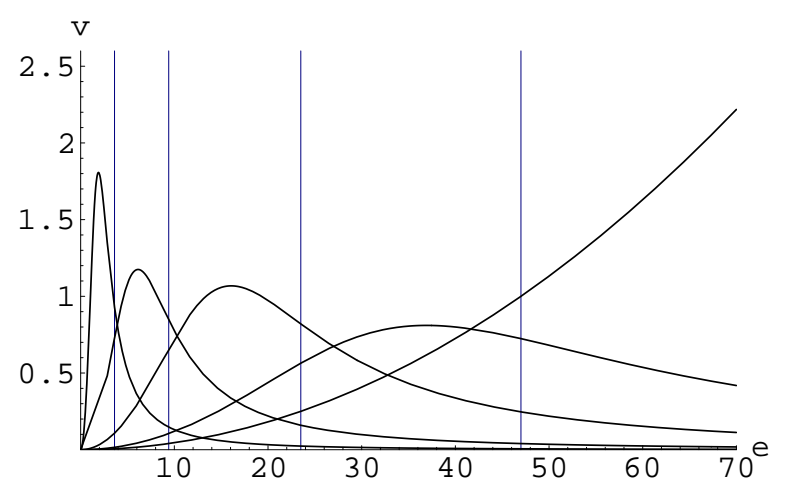

(a)

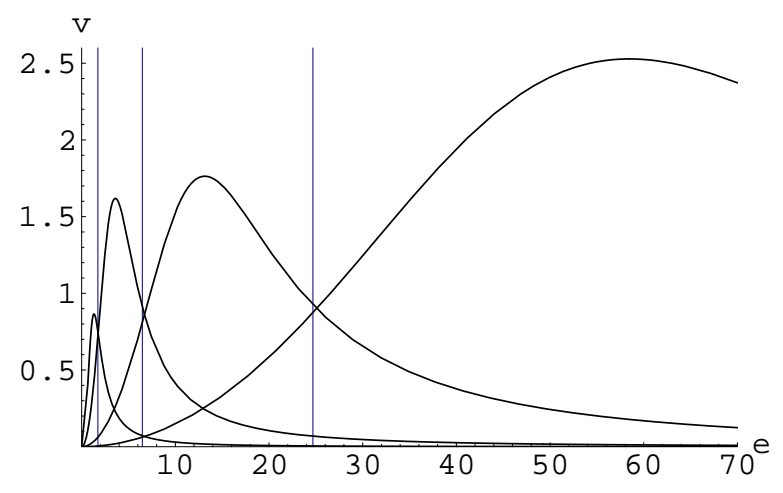

(b)

Figure 3: Phases of Morita equivalent NCSYM for (a) $\Theta=13 / 47$ and (b) $\Theta=\gamma \sim 0.58$. The left most peak illustrates the dependence of the size of the torus as a function of $e$ of the original NCSYM. Each successive peak corresponds to the size of the torus of the Morita dual.

\section{Conclusions}

The goal of the paper was to show that the theories which are related by Morita equivalence have natural bounds in energies (2.3) for which the dynamics of the effective degrees of freedom are most local and therefore natural. In this way, various Morita equivalent theories form a hierarchy of phases, each being the appropriate description for the physics at that scale. We showed, for weakly as well as for strongly coupled theories, that such a natural description is unique at any given energies. For rational $\Theta$ we find a finite chain of natural descriptions which ends in the ultra violet by a twisted OSYM. For irrational $\Theta$ we find an infinite chain of natural NCSYM descriptions.

We will close by commenting on an amusing application ${ }^{3}$ of the NCSYM-OSYM hierarchy to a version of large $N$ reduction of gauge theories $[31,32,33,34]$. As we described earlier,

$$
(U(N) \operatorname{NCSYM} \Theta=1 / s)=(U(s N) \text { OSYM } \tilde{m}=N)
$$

at the microscopic level due to Morita equivalence. The theory on the left hand side can be described to a good approximation by the usual SYM (on the same space-time with the same gauge group and no twist) at energies below the non-commutativity scale

$$
E<\frac{1}{\Delta}=\frac{\sqrt{s}}{\Sigma}
$$

This follows from the fact that the $*$-product deforms the SYM by irrelevant operators which do not affect the infra red dynamics. If we now take the limit $s \rightarrow \infty$, the theory on the

\footnotetext{
${ }^{3}$ Similar ideas were considered in [4].
} 
left hand side reduces to an ordinary $U(N)$ SYM on a torus of size $\Sigma$. On the other hand, the theory on the right hand side becomes a $U(s N)$ theory defined on $R^{2} \times T^{2}$ with a twist $\tilde{m}=N$. The size of the $T^{2}$ goes to zero in the large $s$ limit, and in this way, we have related $U(N)$ theory in four dimensions to a $U(s N)$ theory in two dimensions. It is easy to generalize to a reduction from four dimensions to zero dimensions by considering the case where $x_{0}$ and $x_{1}$ are non-commutative as well. It would be interesting to see if such a relation derived using the Morita equivalence is related to the traditional ideas of large $N$ reduction $[31,32,33,34]$ as well as to some more recent works [35, 36, 37].

\section{Acknowledgments}

We would like to thank D. Gross, K. Hashimoto, B. Pioline, and especially E. Silverstein for stimulating discussions at different stages of the work. The work of AH is supported in part by the National Science Foundation under Grant No. PHY94-07194. The work of NI is supported in part by NSF grant No. PHY97-22022.

\section{Appendix}

In this appendix we will review some basic facts about NCSYM and the Morita equivalence. For concreteness, we will focus on $\mathcal{N}=4 U(N)$ NCSYM on $R \times S_{1} \times T^{2}$ with non-commutative coordinates

$$
\left[x_{\mu}, x_{\nu}\right]=i \theta_{\mu \nu}
$$

We will take $T^{2}$ to be a square torus with radii $\Sigma$, and take the radius $R$ of the $S_{1}$ to be much larger than $\Sigma$. Such a theory is defined by the action

$$
S=\operatorname{Tr} \int d^{4} x\left(\frac{1}{4 g_{Y M}^{2}}\left(F_{\mu \nu}+\Phi_{\mu \nu}\right) *\left(F^{\mu \nu}+\Phi^{\mu \nu}\right)+\ldots\right)
$$

whose parameters, in addition to $\Delta, R$, and $\Sigma$, are the rank of the gauge group $N$, the gauge coupling $g_{Y M}^{2}$ and the background fields $\Phi_{\mu \nu}$. "..." indicates the scalar and the fermion terms. The non-commutativity parameter $\theta_{\mu \nu}$ is encoded in the $*$-product

$$
f(x) * g(x)=\left.e^{\frac{i \theta_{\mu \nu}}{2} \frac{\partial}{\partial x_{\mu}} \frac{\partial}{\partial x_{\nu}^{\prime}}} f(x) g\left(x^{\prime}\right)\right|_{x=x^{\prime}} .
$$

The $\Phi$ dependent term in the action is topological and its main role is to twist [16] the vacuum. This parameter appears in the construction of the NCSYM via the decoupling limit of open strings on D3-branes as was explained in [15]. The precise role played by $\Phi$ in mapping T-duality to Morita equivalence was elucidated recently in [14]. 
We will also restrict our attention to the case where only the 23 component (along the $\left.T^{2}\right)$ of $\theta_{\mu \nu}$ and $\Phi_{\mu \nu}$

$$
\theta_{23}=2 \pi \Delta^{2}, \quad \Phi_{23}=\Phi
$$

is non-vanishing, and introduce a dimensionless non-commutativity parameter

$$
\Theta=\frac{\Delta^{2}}{\Sigma^{2}}
$$

The spectrum of BPS states in this theory was studied extensively $[3,6,7,8,9,10,11,12$, 13, 14]. These states are labeled by the quantum numbers corresponding to the momentum $p_{i}$, the electric flux $w_{i}$, and the magnetic flux $m_{i j}$, and have the masses

$$
E=\frac{\Sigma_{1} \Sigma_{2} \Sigma_{3}}{\Sigma_{i}^{2} \Sigma_{j}^{2}} \frac{\left(m_{i j}+N_{\Theta} \Phi_{i j}\right)^{2}}{4 g_{Y M}^{2} N_{\Theta}}+\frac{\Sigma_{i}^{2}}{\Sigma_{1} \Sigma_{2} \Sigma_{3}} \frac{g_{Y M}^{2}}{2 N_{\Theta}}\left(w_{i}+\Theta_{i j} p_{j}\right)^{2}+\frac{1}{N_{\Theta}} \sqrt{\frac{k_{i}^{2}}{\Sigma_{i}^{2}}},
$$

where

$$
N_{\Theta}=N+\frac{1}{2} m_{i j} \Theta_{j i}, \quad k_{i}=p_{i} N_{\Theta}-m_{i j}\left(w_{j}+\Theta_{j k} p_{k}\right)
$$

$\Sigma_{i}$ is the radius along the $x_{i}$ coordinate, and $i, j, k$ run over $1,2,3$. All of the terms in (A.6) are positive definite. From the first term of (A.6), we can infer that the vacuum (lightest) topological sector is the one with magnetic quantum numbers

$$
m_{12}=m_{13}=0, \quad m=m_{23}=\left\lceil-\frac{N \Phi}{1-\Phi \Theta}\right\rfloor
$$

where $\lceil x\rfloor$ denotes an integer whose value is closest to $x$. In this sector, (A.7) simplifies to

$$
k_{1}=N_{\Theta} p_{1}, \quad k_{i}=p_{i} N-m \epsilon_{i j} w_{j} .
$$

The $k_{i}$ 's label the spectrum of quadratic fluctuations around the background [11]. Note in particular that (A.9) is the defining relation of the Pointing vector in the vacuum $\left(k_{i}=0\right)$.

The energy of an excited state is given in the second and the third terms of (A.6). We will ignore the second term since it only gives rise to a small perturbation when $g_{Y M}$ is small to obtain from the third term

$$
E^{2}=\frac{1}{R^{2}} p_{1}^{2}+\frac{1}{N_{\Theta}^{2} \Sigma^{2}} k_{2}^{2}+\frac{1}{N_{\Theta}^{2} \Sigma^{2}} k_{3}^{2} .
$$

From (A.9) we see that $k_{i}$ takes on values which are integer multiples of $\operatorname{GCD}(m, N)$. These states come with $(\mathrm{GCD}(m, N))^{2}$-fold degeneracy [17] which is a remnant of the $N^{2}$ degeneracy of the gauge particles in the $U(N)$ gauge theory. ${ }^{4}$ Therefore, the entropy is

$$
S=\operatorname{GCD}(m, N)^{2}\left(\frac{N_{\Theta} \Sigma}{\operatorname{GCD}(m, N)}\right)^{2} R T^{3}=N_{\Theta}^{2} \Sigma^{2} R T^{3} .
$$

\footnotetext{
${ }^{4}$ This is closely related to fact that the gauge group of the untwisted NCSYM dual to this theory is $U(\operatorname{GCD}(m, N))$.
} 
Recalling the construction of the NCSYM as a decoupling limit of D3-branes in type IIB string theory wrapping $S_{1} \times T^{2}[1,2,15]$, consider acting on the $T^{2}$ by an element of the T-duality group $S L(2, Z) \times S L(2, Z)$. The full T-duality group survives the decoupling limit and is identified as the group of Morita equivalences of the NCSYM. We will concentrate on $S L(2, Z)$ subgroup which only acts on the Kähler structure. An element of $S L(2, Z)$ will map a NCSYM with rank $N$, twist $m$, coupling $g_{Y M}$, period $\Sigma$, non-commutativity $\Theta$, and background flux $\Phi$, to its Morita equivalent dual with parameters $\tilde{N}, \tilde{m}, \tilde{g}_{Y M}, \tilde{\Theta}$, $\tilde{\Phi}$, and $\tilde{\Sigma}$ according to

$$
\begin{aligned}
& \tilde{\Theta}=\frac{c+d \Theta}{a+b \Theta}, \quad \tilde{\Phi}=(a+b \Theta)^{2} \Phi-b(a+b \Theta), \quad \tilde{\Sigma}=(a+b \Theta) \Sigma, \\
& \tilde{g}_{Y M}^{2}=(a+b \Theta) g_{Y M}^{2}, \quad\left(\begin{array}{c}
\tilde{m} \\
\tilde{N}
\end{array}\right)=\left(\begin{array}{cc}
a & b \\
c & d
\end{array}\right)\left(\begin{array}{c}
m \\
N
\end{array}\right),
\end{aligned}
$$

where $a, b, c$, and $d$ are integers defining the element of $S L(2, Z)$. One can verify that the transformations of $m$ and $\Phi$ are consistent with (A.8). The $S L(2, Z)$ invariance of the entropy (A.11) follows immediately from the transformation of $\Sigma$ and the fact that $N_{\Theta}$ transforms according to

$$
\tilde{N}_{\Theta}=\frac{N_{\Theta}}{(a+b \Theta)}
$$

which implies together with (A.12) that the entropy is indeed invariant. The momentum and the electric flux quantum numbers are also exchanged under the $S L(2, Z)$

$$
\left(\begin{array}{c}
\tilde{p}_{i} \\
\tilde{w}_{i}
\end{array}\right)=\left(\begin{array}{ll}
a & b \\
c & d
\end{array}\right)\left(\begin{array}{c}
p_{i} \\
w_{i}
\end{array}\right) .
$$

Note that a NCSYM with $\Theta=r / s$ for relatively prime integers $r$ and $s$ and $\Phi=0$ is Morita equivalent to an OSYM with

$$
\tilde{\Sigma}=\frac{1}{s} \Sigma, \quad \tilde{N}=s N, \quad \tilde{g}_{Y M}^{2}=\frac{1}{s} g_{Y M}^{2}, \quad \tilde{\Phi}=-\frac{q}{s},
$$

by choosing $c=-r, d=s$, and taking $b=q$ to be the solution of

$$
q r=(1 \operatorname{Mod} s)
$$

The solution of (A.16) is uniquely determined by requiring $q / s$ to take on values between zero and one. For $r=1$, this is solved by $q=1$ which is the case described extensively in sections 2.1 and 2.2. This is the precise statement of equivalence between the NCSYM and the twisted OSYM when $\Theta$ is a rational number. 


\section{References}

[1] A. Connes, M. R. Douglas, and A. Schwarz, "Noncommutative geometry and matrix theory: Compactification on tori," JHEP 02 (1998) 003, hep-th/9711162.

[2] M. R. Douglas and C. Hull, "D-branes and the noncommutative torus," JHEP 02 (1998) 008, hep-th/9711165.

[3] P.-M. Ho, "Twisted bundle on quantum torus and BPS states in matrix theory," Phys. Lett. B434 (1998) 41-47, hep-th/9803166.

[4] D. Bigatti, "Non commutative geometry and super Yang-Mills theory," Phys. Lett. B451 (1999) 324, hep-th/9804120.

[5] G. Landi, F. Lizzi, and R. J. Szabo, "String geometry and the noncommutative torus," Commun. Math. Phys. 206 (1999) 603, hep-th/9806099.

[6] B. Morariu and B. Zumino, "Super Yang-Mills on the noncommutative torus," hep-th/9807198.

[7] C. Hofman, E. Verlinde, and G. Zwart, "U-duality invariance of the four-dimensional Born-Infeld theory," JHEP 10 (1998) 020, hep-th/9808128.

[8] C. Hofman and E. Verlinde, "U-duality of Born-Infeld on the noncommutative two-torus," JHEP 12 (1998) 010, hep-th/9810116.

[9] C. Hofman and E. Verlinde, "Gauge bundles and Born-Infeld on the noncommutative torus," Nucl. Phys. B547 (1999) 157, hep-th/9810219.

[10] A. Schwarz, "Morita equivalence and duality," Nucl. Phys. B534 (1998) 720, hep-th/9805034.

[11] A. Konechny and A. Schwarz, "BPS states on noncommutative tori and duality," Nucl. Phys. B550 (1999) 561, hep-th/9811159.

[12] A. Konechny and A. Schwarz, "Supersymmetry algebra and BPS states of super Yang-Mills theories on noncommutative tori," Phys. Lett. B453 (1999) 23, hep-th/9901077.

[13] A. Konechny and A. Schwarz, "1/4-BPS states on noncommutative tori," JHEP 09 (1999) 030, hep-th/9907008.

[14] B. Pioline and A. Schwarz, "Morita equivalence and T-duality (or B versus Theta)," JHEP 08 (1999) 021, hep-th/9908019. 
[15] N. Seiberg and E. Witten, "String theory and noncommutative geometry," JHEP 09 (1999) 032, hep-th/9908142.

[16] G. 't Hooft, "Some twisted selfdual solutions for the Yang-Mills equations on a hypertorus," Commun. Math. Phys. 81 (1981) 267.

[17] P. van Baal, "SU(N) Yang-Mills solutions with constant field strength on $T^{4}$," Commun. Math. Phys. 94 (1984) 397.

[18] A. Hashimoto and W. Taylor, "Fluctuation spectra of tilted and intersecting D-branes from the Born-Infeld action," Nucl. Phys. B503 (1997) 193, hep-th/9703217.

[19] J. M. Maldacena and L. Susskind, "D-branes and Fat Black Holes," Nucl. Phys. B475 (1996) 679-690, hep-th/9604042.

[20] A. Hashimoto and N. Itzhaki, "Non-commutative Yang-Mills and the AdS/CFT correspondence," Phys. Lett. B465 (1999) 142, hep-th/9907166.

[21] J. M. Maldacena and J. G. Russo, "Large N limit of non-commutative gauge theories," JHEP 09 (1999) 025, hep-th/9908134.

[22] L. Susskind and E. Witten, "The holographic bound in anti-de Sitter space," hep-th/9805114.

[23] N. Itzhaki, J. M. Maldacena, J. Sonnenschein, and S. Yankielowicz, "Supergravity and the large N limit of theories with sixteen supercharges," Phys. Rev. D58 (1998) 046004 , hep-th/9802042.

[24] R. Gregory and R. Laflamme, "Black strings and p-branes are unstable," Phys. Rev. Lett. 70 (1993) 2837, hep-th/9301052.

[25] R. Gregory and R. Laflamme, "The Instability of charged black strings and p-branes," Nucl. Phys. B428 (1994) 399-434, hep-th/9404071.

[26] J. L. F. Barbon, I. I. Kogan, and E. Rabinovici, "On stringy thresholds in SYM/AdS thermodynamics," Nucl. Phys. B544 (1999) 104, hep-th/9809033.

[27] M. Li, E. Martinec, and V. Sahakian, "Black holes and the SYM phase diagram," Phys. Rev. D59 (1999) 044035, hep-th/9809061.

[28] E. Martinec and V. Sahakian, "Black holes and the SYM phase diagram. II," Phys. Rev. D59 (1999) 124005, hep-th/9810224. 
[29] A. Giveon, M. Porrati, and E. Rabinovici, "Target space duality in string theory," Phys. Rept. 244 (1994) 77-202, hep-th/9401139.

[30] J. Maldacena, "The large N limit of superconformal field theories and supergravity," Adv. Theor. Math. Phys. 2 (1998) 231, hep-th/9711200.

[31] T. Eguchi and H. Kawai, "Reduction of dynamical degrees of freedom in the large N gauge theory," Phys. Rev. Lett. 48 (1982) 1063.

[32] G. Bhanot, U. M. Heller, and H. Neuberger, "A phase transition in the quenched Eguchi-Kawai model," Phys. Lett. 115B (1982) 237.

[33] G. Parisi, "A simple expression for planar field theories," Phys. Lett. 112B (1982) 463.

[34] D. J. Gross and Y. Kitazawa, "A quenched momentum prescription for large N theories," Nucl. Phys. B206 (1982) 440.

[35] H. Aoki, N. Ishibashi, S. Iso, H. Kawai, Y. Kitazawa, and T. Tada, "Noncommutative Yang-Mills in IIB matrix model," hep-th/9908141.

[36] I. Bars and D. Minic, "Non-commutative geometry on a discrete periodic lattice and gauge theory," hep-th/9910091.

[37] J. Ambjorn, Y. M. Makeenko, J. Nishimura, and R. J. Szabo, "Finite N matrix models of noncommutative gauge theory," hep-th/9911041. 